Aber weder im Zentralkomitee noch im Politbüro dachte die Mehrheit jemals daran, Sinowjew zum Führer zu erheben, da diese Führerrolle die Beherrschung des Parteikongresses zur Voraussetzung hatte. Und hier hatte Stalin das Heft in der Hand. Langsam brachte er seine Leute überall als Sekretäre in der Parteiorganisation unter und schreckte vor nichts zurück, um die Kontrolle des Parteiapparates in seine Hand zu bekommen. ${ }^{386}$

Aber auch wenn die Mitglieder des Politbüros mehrheitlich gegen Trotzki eingestellt waren und nicht daran dachten, Sinowjew zum späteren Nachfolger Lenins zu machen, war zum Zeitpunkt der Krankheit Lenins keineswegs klar, dass mehrere Jahre danach Stalin zum Alleinherrscher avancierte. Denn als Lenin am 21. Januar 1924 in seinem Landhaus in Gorki in der Nähe Moskaus verstarb, waren zweifelsohne »viele Wege« (Arendt) möglich.

\title{
2.3 Der Aufstieg Stalins und die "Zweite Revolution« Ende der 1920er-Jahre
}

Beim Tod Lenins war seine Nachfolge nicht genau geregelt. Allerdings lag das »Politische Testament « vom 25. Dezember 1922 vor, das seine Ansicht über die oberste Parteiführung in einer Übergangsform zum Ausdruck brachte. Angesichts der Gefahr einer möglichen Parteispaltung, die Lenin insbesondere aufgrund der starken Rivalität zwischen Stalin und Trotzki befürchtete, favorisierte er implizit eine kollektive Führung. Darüber hinaus bezeichnete er Bucharin einerseits als den »wertvollsten Theoretiker der Partei« und andererseits als den »Liebling der ganzen Partei«. In dem »Nachtrag《 vom 4. Januar $1923 \mathrm{zu}$ seinem »Testament" sprach sich Lenin auch angesichts von Stalins negativen Charaktereigenschaften klipp und klar für dessen Entfernung vom Posten des Generalsekretärs aus. Am 5. März 1923 brachte er Stalin persönlich in einem Schreiben das Ende der "persönlichen und kameradschaftlichen Beziehungen« zum Ausdruck. Genau schrieb Lenin in seinem »Politischen Testament«, das von David Shub in seinem Beitrag Lenins letzte Tage im Monat veröffentlicht wurde:

Genosse Stalin hat, nachdem er Generalsekretär geworden war, ungeheure Macht in seinen Händen vereinigt, und ich bin nicht sicher, ob er es immer versteht, von dieser Macht mit genügender Behutsamkeit Gebrauch zu machen. Andererseits zeichnet sich Cenosse Trotzki, wie sein Kampf gegen den Zentralausschuß im Zusammenhang mit der Frage des Volkskommissariats für das Verkehrswesen bewiesen hat, nicht nur durch außergewöhnliche Fähigkeiten aus - er ist sicherlich der fähigste Kopf im gegenwärtigen Zentralkomitee -, sondern auch durch übermäßiges Selbstvertrauen und durch die Neigung, sich zu sehr der rein administrativen Seite der Regierungsgeschäfte zu widmen.

Diese beiden Eigenschaften der beiden fähigsten Führerpersönlichkeiten im gegenwärtigen Zentralkomitee könnten ganz unwillkürlich zu einer Spaltung führen. Wenn unsere Partei keine Maßnahmen zu ihrer Verhinderung ergreift, könnte sie ganz unerwartet eintreten.

386 David Shub, Lenins letzte Tage. Aus einer neuen kritischen Biographie, in: Der Monat 1 (1948), H. 3, S. 45 . 
Ich habe nicht die Absicht, die übrigen Mitglieder des Zentralausschusses hinsichtlich ihrer persönlichen Eigenschaften zu charakterisieren. Ich will sie nur daran erinnern, daß der Zwischenfall mit Sinowjew und Kamenew im Oktober selbstverständlich nicht zufällig war, daß man ihn aber ebensowenig nachtragen sollte wie Trotzki seinen »Nichtbolschewismus«.

Was die jungen Mitglieder des Zentralkomitees anbelangt, so möchte ich über Pjatakow und Bucharin ein paar Worte sagen. Nach meiner Meinung sind sie die fähigsten Köpfe (unter der jungen Ceneration). Dabei darf man aber folgendes nicht vergessen: Bucharin ist nicht nur der wertvollste Theoretiker der Partei - und zugleich der umfangreichste -, sondern auch als Liebling der ganzen Partei zu betrachten. Man kann seine theoretischen Ansichten aber nur mit den größten Vorbehalten als völlig marxistisch ansehen, denn es steckt etwas vom Scholastiker in ihm. (Er hat die dialektische Methode nie gelernt und vermutlich nie ganz erfaßt.) Pjatakow endlich ist ein Mann, der sich zweifellos durch Wollen und Fähigkeit auszeichnet, sich aber zu sehr der administrativen Seite der Arbeit widmet, als daß man sich in ernsten politischen Fragen auf ihn verlassen könnte. Selbstverständlich gelten diese beiden Bemerkungen lediglich für den Augenblick oder unter der Voraussetzung, daß diese beiden fähigen und treuen Arbeiter sich nicht veranlaßt sehen sollten, ihr Wissen zu ergänzen und sich von ihrer Einseitigkeit frei zu machen.

25. Dezember 1922

Nachtrag: Stalin ist zu grob, und dieser Fehler, über den man unter uns Kommunisten vollkommen hinwegsehen könnte, wird bei der Stellung des Ceneralsekretärs unerträglich. Deshalb schlage ich den Genossen vor, einen Weg zur Entfernung Stalins aus dieser Stellung zu finden und einen anderen Mann zu ernennen, der Stalin an Energie gleichkommt, sonst aber im Wesen von ihm verschieden ist, nämlich geduldiger, treuer, höflicher und den Cenossen gegenüber aufmerksamer, weniger launisch usw. Dieser Umstand mag als unwesentliche Kleinigkeit erscheinen, aber ich glaube, daß er zur Vermeidung einer Spaltung und mit Rücksicht auf das Verhältnis zwischen Stalin und Trotzki, von dem ich oben gesprochen habe, keine Kleinigkeit, sondern von entscheidender Bedeutung ist.

4. Januar 1923 Lenin. ${ }^{387}$

Nach dem Tod von Lenin kam es zu einer Veränderung im Herrschaftsgefüge des bolschewistischen Apparates. A. I. Rykow übernahm den Vorsitz im Rat der Volkskommissare und Kamenew wurde zum Vorsitzenden im Politbüro ernannt. Die eigentliche Macht schien weiterhin in den Händen der Troika zu liegen.

Gleichwohl verschärften sich allmählich die politischen Auseinandersetzungen in der Führungsgruppe der Partei. Trotz der bestehenden persönlichen Ressentiments der Rivalen ging es indes in erster Linie um die Verwirklichung verschiedenartiger politisch-ideologischer Konzepte innerhalb der Partei. Angesichts der weltpolitischen Lage entwickelte Stalin seine Theorie vom »Sozialismus in einem Land«, die er im Frühjahr 1925 auf einer Parteikonferenz vorlegte. Auf der anderen Seite vertrat Trotzki weiterhin den Standpunkt "permanente Revolution", und das bedeutete, dass ohne eine Revolutionierung der westlich-kapitalistischen Staaten der sogenannte wirkliche 
Sozialismus in Russland nicht verwirklicht werden könne; er verkörperte den Führer des linken Flügels der Partei. Die beiden einflussreichen "Altbolschewiken« Sinowjew und Kamenew favorisierten einen ähnlichen »internationalistischen« Kurs, betrieben allerdings gleichzeitig eine undurchsichtige Machtpolitik zwischen den beiden Hauptkontrahenten und "Todfeinden" Trotzki und Stalin. Zudem existierte innerhalb der Partei noch ein »rechter Flügel«, der von Bucharin und dem Gewerkschaftsführer Tomski repräsentiert wurde und der für einen »liberalen Kurs« insbesondere gegenüber der Bauernschaft plädierte. Gleichwohl zeichnete sich ab, dass fortan die eigentliche Kontroverse zwischen Trotzki und Stalin ausgetragen würde, die die zwei entscheidenden unterschiedlichen ideologischen Richtungen vertraten. In letzter Konsequenz ließ es indes Trotzki an der absoluten Bereitschaft vermissen, mit Überzeugung für seine politisch-ideologische Konzeption einzutreten und am Ende möglicherweise die politische Führung in der Partei zu übernehmen. Anders gesagt: $\mathrm{Zu}$ einer Rebellion gegen die Parteiführung im Sinne eines bonapartistischen Schritts konnte er sich nicht entschließen und war viel zu sehr in seinem deterministischen Revolutionskonzept gefangen. Anfang 1925 wurde Trotzki als Kriegskommissar abgesetzt, und bevor weitere Maßregelungen vorgenommen wurden und die Troika eine gemeinsame Führungspolitik betreiben konnte, zerfiel sie bereits.

Ein wichtiges Ereignis stellte der XIV. Parteikongress im Dezember 1925 dar, auf dem Stalin mit seinem Industrialisierungsprogramm in Erscheinung trat und hierbei von Sinowjew und Kamenew kritisiert wurde. Indessen konnten sie sich mit ihren Absichten nicht durchsetzen, und Stalin gelang es bei der Abstimmung, eine überwältigende Mehrheit zu erzielen. Stalin nutzte seine gestärkte Position in der Partei, um 1926 Sinowjew aus seiner einflussreichen Funktion als Parteisekretär von Leningrad $\mathrm{zu}$ entlassen und durch den eine "neue« Generation von Bolschewiki verkörpernden, ihm loyal verbundenen S. M. Kirow zu ersetzen. Daraufhin kam es zu einer Annäherung zwischen Sinowjew und Trotzki, der jetzt bereit war, den Kampf um die Macht mit Stalin aufzunehmen. Indes: Stalins Stellung in der Partei war bereits zu diesem Zeitpunkt so stark, dass er imstande war, Sinowjew (und dessen Kampfgefährten Kamenew) aus sämtlichen Ämtern zu verdrängen. Als im Sommer 1927 die Position Stalins aufgrund seiner außenpolitischen Misserfolge angesichts der »China-Frage« geschwächt schien, unternahm der »Block« Trotzki, Sinowjew und Kamenew auf dem XV. Parteikongress im Dezember 1927 den Versuch, verlorenes politisches (Macht-) Terrain zurückzugewinnen. Kamenew plädierte in seiner Rede für das Recht auf Kritik an der Parteiführung und meinte selbstverständlich Stalin, der wiederum den entfachten heftigen Debatten ein Ende bereitete. Nach dem Parteikongress wurden zahlreiche führende Funktionäre aus der Partei ausgestoßen. Trotzki musste zunächst in die Verbannung nach Alma-Ata (und wurde schließlich im Januar 1929 aus der Sowjetunion ausgewiesen), und Kamenew sowie Sinowjew mussten nach einem "formellen Beschluss« die Partei verlassen. Gleichwohl war Stalin zu diesem Zeitpunkt noch nicht imstande, über die Geschicke der Partei alleine zu entscheiden. So musste er es hinnehmen, dass nach Ablauf der Frist am 1. Juli 1928 mit Sinowjew und Kamenew seine »beiden gefährlichen Feinde als vollberechtigte Mitglieder wieder in die Partei« aufgenommen wurden. ${ }^{388}$

388 Ruth Fischer, Tito und Trotzki. Der Unabhängigkeitskampf der kommunistischen Parteien, in: Der Unabhängigkeitskampf der kommunistischen Parteien, in: Der Monat 2 (1950), H. 16, S. 400. 
Gleichwohl sollten Kamenew und Sinowjew fortan keine Rolle mehr spielen und wurden von Stalin »kaltgestellt«. Trotzki wiederum musste konstatieren, dass er den politischen Machtkampf verloren hatte, und mithin hatte Stalin den zweifelsohne gefährlichsten Konkurrenten ausschalten können. Mit der Frage, warum in diesem »Bruderkampf« Stalin und nicht Trotzki als Sieger hervorging und die für den weiteren Verlauf des sowjetkommunistischen Herrschaftssystems von zentraler Bedeutung sein sollte, beschäftigte sich der Autor der 1948 erschienenen wichtigen Studie Three who made a Revolution, Bertram D. Wolfe, im Monat anlässlich seiner Besprechung des "Stalin-Buches« von Leo Trotzki. ${ }^{389}$ Auf dem Hintergrund von Reflexionen über Stalins »Stärken ${ }^{390}$ stellte Wolfe zu Recht die sich im Nachhinein aufdrängende Frage: »Wie konnte ein Mensch von so unheilbarer `Mittelmäßigkeit die größte persönliche Machtstellung auf Erden erlangen?« Hierzu schrieb er mit Blick auf die Ausführungen von Trotzki in diesem Zusammenhang:

Mit Recht sucht Trotzki eine Begründung hierfür nicht auf psychologischem, sondern auf soziologischem Cebiet. Aber auch seine soziologischen Thesen hemmen die Analyse mehr, als daß sie ihr Richtung geben. In den Augen Trotzkis wäre die »Reaktion nach dem Muster des Thermidor «nur durch die Ausbreitung der Revolution nach Westen zu vermeiden gewesen. Stalin war »der bestmögliche Ausdruck dieser bürokratisch-konservativen Reaktion « und sein Aufstieg zur Macht somit unvermeidlich. [...] Wie kann man jedoch die Reaktion bekämpfen, wenn man von dem Crundsatz ausgeht, daß sie unvermeidlich ist? Wie kann man die Kräfte moralischer Verdammung gegen ein Phänomen ins Feld führen, das in seiner naturhaften Unabwendbarkeit einem Erdbeben gleicht? Offensichtlich ist dies einer der geheimen Cründe dafür, daß Trotzki in dem Kampf mit seinem Cegenspieler unterlag [...].

Für Wolfe stand fest, dass Trotzki im Kontext der Oktoberrevolution nach seinem Dafürhalten - im Unterschied zu Stalin, der zu diesem Zeitpunkt und in den Jahren danach den russischen »Massen« unbekannt blieb - mit seiner »flammenden Beredsamkeit die Massen aufzurütteln« verstand, mithin sozusagen agitatorische Qualitäten hatte und zudem intellektuelle und schriftstellerische Fähigkeiten besaß. »In dieser Hinsicht«, so Wolfe,

war und blieb Stalin bis zum heutigen Tage, was Trotzki in ihm sieht: eine unscheinbare und farblose Mittelmäßigkeit. Als Konstrukteur eines politischen Apparats jedoch oder wenigstens als Meister der Kunst, einen solchen Apparat bei passender Gelegenheit für sich zu gewinnen und den eigenen Zwecken dienstbar zu machen, wie in der Handhabung von Menschen und in der vollendeten Sicherheit, mit der er seine Kräfte ansetzt, können sich nur wenig mit Stalin messen. Und Trotzki gehörte sicher nicht dazu. So-

389 Bertram D. Wolfe, Trotzki über Stalin, in: Der Monat 1 (1948), H. 3, S. $106-111$.

390 Beispielsweise schrieb Wolfe hierzu (ebd., S. 108): »Manchmal zögernd, dann wieder sehr großzügig, bemüht sich Trotzki, soweit wie möglich Stalins Stärken anzuerkennen: sunbezähmbarer Wille (eine Willenskraft, die seine geistigen Fähigkeiten immer weitaus übertraf), Charakterfestigkeit, entschlossene Handlungsweise, Zähigkeit, Eigensinn und bis zu einem gewissen Crade sogar Verschlagenheit [...], Rücksichtslosigkeit und die Neigung zu Komplotten, Eigenschaften, die im Kampf gegen eine feindliche Klasse unentbehrlich sind, persönlicher Mut, kalte Beharrlichkeit und ein praktischer, gesunder Menschenverstand.« 
lange er von Lenin getrennt war, erwies sich Trotzki stets als unfähig, einen politischen Apparat zu schaffen. Als er zu einem Teil des Leninschen Apparates geworden war, verstand er es nicht, davon Besitz zu nehmen. ${ }^{391}$

Nachdem Stalin seinen für ihn gefährlichsten politischen Gegenspieler ausgeschaltet hatte, startete er den nächsten Angriff. In nicht minderer Form ging er gegen Bucharin, Rykow und Tomski vor, die im Jahre 1929 ihre Parteiämter verloren, sodass Stalin sich nach der Ausschaltung sämtlicher potenzieller Kontrahenten auf dem »besten« Weg zum uneingeschränkten Führer des bolschewistischen Regimes befand. Zu diesem Zeitpunkt konnte Stalin noch keineswegs schalten und walten, wie es ihm beliebte, denn noch existierten oppositionelle Gruppen in der bolschewistischen Partei. Anders gesagt: Die bolschewistische Partei stellte noch keineswegs ein monolithisches Gebilde dar. Die Ausschaltung der sogenannten rechten Opposition um Bucharin stand im Zusammenhang mit dem auf dem XIV. Parteitag im Dezember 1925 von der politischen Führung des sowjetischen Staates beschlossenen und von Stalin favorisierten Industrialisierungsprogramm, dem dann im Jahre 1929 die »Zweite Revolution«, d.h. die sogenannte Kollektivierungspolitik, folgen sollte.

Vor dem Hintergrund der im Dezember 1924 verkündeten Doktrin Stalins vom »Aufbau des Sozialismus in einem Land« beschloss der XV. Parteitag im Dezember 1927 einen Wirtschaftsplan (erster Fünfahresplan), demzufolge die Sowjetunion aus einem Agrarland in ein Industrieland verwandelt werden und der Anfang 1928 beginnen sollte. Während sich in den Jahren 1924 bis 1927 die Lage der Bauern positiv entwickelte, mehrten sich zu diesem Zeitpunkt die Anzeichen dafür, dass die industriellen Wachstumsraten hinter den gesamtwirtschaftlichen Anforderungen zurückblieben und mithin die Ziele der bolschewistischen Führungsspitze nicht erreicht würden; zudem verschlechterte sich die Versorgungssituation in den Großstädten. Bucharin, Tomski und Rykow als Vertreter der »Partei-Rechten« sprachen sich für eine höhere Besteuerung der Kulaken und eine staatliche Vorratsbildung (ohne in das gescheiterte Requisitionssystem des »Bürgerkriegskommunismus« zurückzufallen) aus und plädierten dafür, auf die Lage der Bauern insgesamt Rücksicht zu nehmen und das Industrialisierungstempo zurückzuschrauben. Stalin wiederum wollte das bereits von Lenin ins Auge gefasste Programm einer Kollektivierung der Landwirtschaft zum Leben erwecken und es zu einer Zwangskollektivierung steigern, um somit die Industrialisierung des Landes zu forcieren. Er verkündete im Dezember 1929 die »Zweite Revolution" - eine Revolution, die eine Bedeutung wie die Oktoberrevolution haben sollte. »Von oben« sollte auf Initiative des Staates die »Liquidierung der Kulaken als Klasse und die Zwangskollektivierung der gesamten sowjetischen Landwirtschaft innerhalb der nächsten Jahre vollzogen werden. »Die reichen Bauern«, so Löwenthal, »die Kulaken, lösten die Großgrundbesitzer und Kapitalisten als zu liquidierende >feindliche Klasse $a$ ab. ${ }^{392}$

Das Leben von schätzungsweise 100 Millionen Bauern veränderte sich radikal. Der Widerstand des »besitzenden Bauerntums « angesichts der wirtschaftlichen Existenzgefährdung und der damit verbundenen Gefahr der physischen »Vernichtung« wurde

391 Ebd., S. 109.

392 Richard Löwenthal, Ideologie und Realpolitik. Glauben die Kreml-Machthaber an Dogmen?, in: Der Monat 10 (1958), H. 117, S. 37-46, hier S. 45. 
von Stalin gnadenlos durch die GPU bekämpft. ${ }^{393}$ Bis zu schätzungsweise sechs Millionen »Kulaken « wurden entweder in ein Zwangsarbeitslager geschafft (und fanden dort den Tod) oder nach Sibirien deportiert. ${ }^{394}$ Ein besonders verheerendes Ausmaß nahm die sogenannte Zweite Revolution, d.h. die Politik der Zwangskollektivierung der Landwirtschaft, von Stalin in der Ukraine an. Im Jahre 1932/33 weigerten sich die Bauern, der Aufforderung nachzukommen, in die kollektiven Großbetriebe (Kolchosen) einzutreten, und antworteten mit einer Welle von Sabotage- und Widerstandshandlungen. Die Bauern schlachteten ihr Vieh, verbrannten oder versteckten ihre Ernte. Daraus entwickelte sich eine Hungerskatastrophe im ganzen Land. Allein in der Ukraine starben nach Angaben von Koestler schätzungsweise bis zu zwei Millionen Menschen. ${ }^{395}$ Insgesamt starben in der Sowjetunion infolge der Hungersnot nach damaligen Schätzungen etwa zwischen fünf bis acht Millionen Menschen. ${ }^{396}$

In dem Beitrag Churchill und Stalin ging Lasky im Monat u. a. auf die Zwangskollektivierungspolitik Stalins ein. Lasky berichtete davon, wie der Kremlführer während eines Gespräches über die mögliche Nachkriegsordnung nach einem alliierten Sieg gegen »Hitlerdeutschland« mit dem englischen Premierminister Churchill in Moskau im Jahre 1942, auch die politischen Hintergründe der »Kolchosenpolitik« der sowjetischen Führung zur Sprache brachte; die laut Stalin mit einem "furchtbaren Kampf« verbunden war. In diesem Kontext zitiert Lasky folgende Passage aus den Memoiren Churchills, die aus der Unterredung mit Stalin stammt und die nicht zuletzt ein bemerkenswertes Zeugnis von Stalins Charakter und dessen »revolutionärer« Moral darstellt, denn es ging immerhin um Millionen von Toten und um Millionen deportierter Menschen:

393 Franz Borkenau, Stalin im Schafspelz. Zu einer neuen Biographie von Isaac Deutscher, in: Der Monat 2 (1949), H. 14, schrieb in diesem Zusammenhang auf S. 206: »Die sSchere bestand in dem immer schärferen Auseinandergehen von Industrie- und Agrarpreisen zu ungunsten der letzteren; die Bauern konnten schließlich für den Erlös ihrer Produkte so gut wie nichts mehr kaufen. Eben dagegen rebellierten die Bauern letzten Endes mit dem Lieferstreik, den sie mit dem Verlust des Eigentums an ihrem Land und mit Millionen von Toten und Verschleppten büßen mußten.«

394 Michael Padev, Die Bauern unter dem Sowjetsystem, in: Der Monat 4 (1951), H. 39, S. 327.

395 Arthur Koestler, Sowjet-Mythos und Wirklichkeit, in: Ders., Der Yogi und der Kommissar, Eßlingen a. N. 1950, S. 248. Vgl. auch den persönlichen Erfahrungsbericht angesichts der Hungersnot in der Ukraine bzw. in Charkow anlässlich einer Reise von Koestler unter der Überschrift »Mißtrauen, Terror und Apathie«: Koestler, Verlorene Illusionen, S. 160 f. Zur Hungersnot in der Ukraine 1932/33 siehe auch den Hinweis von Ivan L. Rudnytsky, Anti-sowjetisch oder anti-russisch, in: Der Monat 2 (1950), H. 19, S. 104-107, auf S. 104, der für das Jahr 1933 von »mehreren Millionen« Toten in der »Ukraine, im Don-Cebiet und Nordkaukasus«sprach, die durch die »künstliche Hungersnot« infolge der »von Moskau angewandte[n] Maßnahme zur Niederringung der widerspenstigen Bauernschaft« ums Leben kamen.

396 Dallin/Nicolaevsky, Arbeiter oder Ausgebeutete, S. 100. Michael Padev (Die Bauern unter dem Sowjetsystem, in: Der Monat 4 [1951], H. 39, S. 327) schrieb in seinem Beitrag, einem Leserbrief, angesichts des Problems der Überprüfbarkeit der Opferzahlen: »Die Sowjetbehörden haben nie irgendwelche Statistiken über die Sterblichkeit der Landbevölkerung während dieser Jahre veröffentlicht, und wir werden wahrscheinlich niemals erfahren, wie viele Bauern im Laufe der Kollektivierungskampagne ums Leben kamen. Ehemalige kommunistische Beamte sprachen davon, daß fünf bis acht Millionen Bauern allein verhungert seien; andere zuverlässige Quellen geben mehr als zehn Millionen an. In jedem Fall ist die Zahl der Toten größer als die Gesamtverluste der Roten Armee während des letzten Krieges, die auf etwa sieben Millionen geschätzt werden.« 
»Das habe ich mir schon gedacht«, sagte Churchill, der Aristokrat und Grundbesitzer, »denn Sie hatten es ja nicht nur mit einigen tausend Aristokraten und Großgrundbesitzern zu tun, sondern mit Millionen von Kleinbauern."

»Zehn Millionen«, erwiderte Stalin und hob die Hände empor. [...] »Es war furchtbar. Vier Jahre dauerte es. Aber es war für Rußland unbedingt erforderlich, mit Traktoren zu pflügen, wenn wir periodisch wiederkehrende Hungersnöte vermeiden wollten. Wir mußten unsere Landwirtschaft mechanisieren. Als wir den Bauern Traktoren gaben, waren diese in wenigen Monaten restlos ruiniert, nur die mit Reparaturwerkstätten konnten mit Traktoren umgehen. Wir haben uns die größte Mühe gegeben, dies den Bauern klarzumachen. Aber es war nicht mit ihnen zu reden. Wenn man einen solchen Bauern mit allen Mitteln zu überzeugen versucht hat, erklärt er, er müsse nach Hause gehen und seine Frau und seinen Hirten um Rat fragen. Schließlich aber läuft die Antwort immer wieder darauf hinaus, daß er nicht in die Kolchose eintreten und daher lieber auf einen Traktor verzichten will.«

»Das waren also Ihre sogenannten Kulaken?«

»]a«, antwortete Stalin. Aber, so stellt Churchill ausdrücklich fest, er wiederholte das Wort »Kulak« nicht. Nach einer langen Pause: »Es war alles sehr traurig und schwierig aber notwendig."

»Was geschah?«, fragte Churchill, als ob er es nicht wußte.

»Nun«, antwortete Stalin, »viele schlossen sich uns an. Anderen wurde in den Provinzen Tomsk oder Irkutsk oder noch weiter im Norden eigenes Land gegeben, aber der größte Teil war so unbeliebt, daß sie von ihren Arbeitern beseitigt wurden [...].«

In diesem Zusammenhang kam Lasky auf die nachträglichen Reflexionen von Churchill zu sprechen:

Churchill spricht heute von »dem starken Eindruck, den mir der Gedanke an die Liquidierung oder Umsiedlung von Millionen bereitete. Ohne Zweifel würde einmal eine Ceneration heranwachsen, der alle diese Greuel unbekannt sind, die aber mehr zu essen haben und dafür Stalins Namen preisen wird.«Ich vermochte nicht, Burkes Ausspruch zu wiederholen: »Wenn ich keine Reform ohne Unrecht haben kann, so verzichte ich auf sie.«Angesichts des großen Krieges, in den wir alle verstrickt waren, schien es mir sinnlos, meine Gedanken über Moral zu äußern. ${ }^{397}$

Die im Zuge der stalinschen »Zweiten Revolution« durch das bolschewistische Regime verhafteten Kulaken bildeten auch und vor allem ein wichtiges Reservoir an Arbeitskräften im Kontext der forcierten Industrialisierung des Landes. Denn sie bildeten Anfang der 1930er-Jahre als verhaftete "Klassenfeinde« die Hauptgruppe, die in den Gefängnissen und Lagern Zwangsarbeit vollrichten mussten. Die Zahl der Insassen war bereits im Verlauf der 1920er-Jahre sukzessive gestiegen. Mit Beginn des Ersten Fünfjahresplanes, genauer mit Beginn der Massenkollektivierung im Jahre 1930, veränderte sich der Charakter der Gefängnisse und der Konzentrationslager, denn in dieser Zeit wurde von der sowjetischen Führung das »System der Arbeitsbesserungslager« konstituiert. In ihrer 1948 erschienenen Studie Arbeiter oder Ausgebeutete. Das

397 Melvin ]. Lasky, Churchill und Stalin. Ein Streifzug durch Winston Churchills Kriegserinnerungen, in: Der Monat 4 (1951), H. 38, S. 141-154, hier S. 153 (Rezension). 
System der Arbeitslager in Sowjetrußland schätzten Dallin und Nikolajewski, dass sich die Gesamtzahl der Gefangenen, die sich 1930 in den sechs im Norden des Landes gelegenen Arbeitslagern befanden, auf schätzungsweise 666257 belief. In den beiden Jahren danach, also 1931 und 1932, betrug die Gesamtzahl bereits zwei Millionen, die sich in den »Haftstätten « befanden. ${ }^{398}$

Bereits in der ersten Ausgabe des Monat im Oktober 1948 wurde die Studie von Dallin und Nikolajewski rezensiert und auf ihre sowohl wissenschaftliche als auch politische Bedeutung insistiert, obwohl zu diesem Zeitpunkt das »Archiv-Problem« existierte. Shub schrieb:

Das Buch liefert mit seiner Fülle von Zitaten, Augenzeugenberichten und photokopierten Dokumenten aus NKWD-»Korrektions-Arbeitslagern «erschöpfendes Material für ein gründliches Studium des Problems. [...] Dallin und Nicolaevsky verhalten sich gegenüber dem System, das sie beschreiben, keineswegs »objektiv«. Sie haben das Cefühl für das Schandbare nicht verloren. Trotz dieses durchaus begrüßenswerten Vorurteils haben sie aber beim Zusammensetzen des äußerst komplizierten TatsachenMosaiks hervorragende Arbeit geleistet, wenn auch noch einige Lücken bleiben. Sie zu schließen, wird dem Historiker vorbehalten bleiben, dem später einmal sämtliche NKWD-Archive zugänglich sind. ${ }^{399}$

Dallin und Nikolajewski konnten bereits 1948 zeigen, dass mit der Einführung des ersten Fünfjahresplanes im Jahre 1928 und dem In-Gang-Kommen des Kollektivierungsprogramms von Stalin 1930, d.h. der »Zweiten Revolution«, nicht nur sich in der sowjetischen Innenpolitik eine radikale Veränderung ergab, sondern sich auch der Charakter der russischen Gefängnisse und Lager verwandelte und die Zahl der Inhaftierten durch ein schnelles Wachstum gekennzeichnet war. Bis zu diesem Zeitpunkt, d.h. in den Jahren nach der Russischen Revolution, ging es mit dem Gefängnissystem, das dem Volkskommissariat der Justiz unterstand, darum, "gewöhnliche Kriminelle« $\mathrm{zu}$ inhaftieren. Im Vordergrund stand der Gedanke, die Inhaftierten zu »bessern « und die Gefängnisse sollten sich selbst finanzieren. ${ }^{400}$ Auf der anderen Seite existierten seit Oktober 1917 Konzentrationslager und Sondergefängnisse des russischen Geheimdienstes (Tscheka bzw. GPU) unter der Leitung von Feliks Dzierzynski (Volkskommissar für Innere Angelegenheiten), die eine genuine politische Bedeutung besaßen. Inhaftiert wurden die politischen Gegner bzw. die politischen »Feinde« des bolschewistischen Regimes, deren Zahl im Laufe der 1920er-Jahre anstieg. Ab 1923 richtete der russische Geheimdienst die »Nördlichen Lager zur besonderen Verwendung« ein. Sie bildeten die eigentliche Keimzelle des zukünftigen Arbeitslagersystems des sowjetischen Staates. Ebendiese Lager befanden sich im Norden des Landes auf

398 Dallin/Nicolaevsky, Arbeiter oder Ausgebeutete, S. 11.

399 Boris Shub, Lüge und Illusion, in: Der Monat 1 (1948), H. 1, S. 88-90 (Rezension). Zur grundsätzlichen Bedeutung der sowjetischen Lager und speziell der ökonomischen Funktion sowie in diesem Zusammenhang der Rolle der Zwangsarbeit in Bezug auf die Genesis des sowjetischen Staates vgl. die entsprechenden Ausführungen von Drew Middleton, Sowjet-Russland ohne Propaganda. Bericht eines Auslands-Korrespondenten, in: Der Monat 1 (1948), H. 1, S. 27-43.

400 Dallin/Nicolaevsky, Arbeiter oder Ausgebeutete, S. 69 f., kamen zu dem Schluss, dass die Zahl der Insassen von 24095 im Jahre 1917 auf 198000 Anfang 1927 anstieg; damit war die Zahl der Gefängnisinsassen in etwa so hoch wie am »Vorabend« der Revolution. 
den Solowezki-Inseln im Weißen Meer. Nach Schätzungen von Dallin und Nikolajewski gab es zum Beispiel in dem zentralen Konzentrationslager der GPU, im Lager Solowezki, im Jahre 19234000 Gefangene. Die Gesamtanzahl der Gefangenen der »Nördlichen Lager« betrug 1 925, also zwei Jahre später, 7 000. In den Jahren 1929 und 1930 wuchs die Zahl der Gefangenen auf über $100000 .{ }^{401}$

Im Hinblick auf die Häftlingskategorien für das Lager Solowezki stellten die beiden Autoren fest:

Drei Gefangenengruppen konstituierten zu jener Zeit die hauptsächliche Bevölkerung des Lagers: 1. gewöhnliche Verbrecher des Typs, der als unverbesserlich angesehen wurde; 2. »Konterrevolutionäre«, gewöhnlich als »K-Rs« bekannt, und 3. »Politische«. [...] Die Unterscheidung zwischen Konterrevolutionären und Politischen hatte man aus dem vorrevolutionären Ringen und aus dem Jahre 1917 geerbt. Anhänger und Führer früherer Rechtsparteien, Anhänger des vorrevolutionären Regimes, die gemäßigt-liberale Partei der Konstitutionellen Demokraten, Kommandeure der Weißen Armee und ähnliche Gruppen fielen unter die umfangreiche Kategorie der Konterrevolutionäre. Auf der anderen Seite wurden Sozialdemokraten, sämtliche Parteien der Populisten, Sozialistische Revolutionäre, Linkssozialistische Revolutionäre und Anarchisten als »antisowjetische« Elemente und ihre Mitglieder in den Arbeitslagern als Politische bezeichnet. $^{402}$

Obwohl die Gefangenen in den GPU-Lagern Strafarbeiten verrichten mussten, damit sich die einzelnen Lager selbst finanzieren konnten, dominierte in den 1920er-Jahren an diesen Orten der sogenannte Strafcharakter. Denn in diesen Lagern war das primäre Ziel des sowjetischen Staates, durch die GPU die Persönlichkeit der Insassen zu zerstören. Die "große Umwälzung « für die Konzentrationslager der GPU kam Ende der 1920er-Jahre. Im Zuge der forcierten Industrialisierung des Landes und der radikalen Kollektivierungspolitik unter Stalins Führung wurde das "System der Arbeitsbesserungslager« ins Leben gerufen. Fortan sollten nach einem Erlass der Regierung die meisten Gefängnisinsassen in den Arbeitslagern Strafarbeit leisten. Anders gesagt, jede Gefängnisarbeit - auch in den Gefängnissen, die noch dem Justizkommissariat unterstanden - avancierte zum Gegenstand der wirtschaftlichen Planung. Die entscheidende Entwicklung begann allerdings, als in diesem Zusammenhang die GPU 1930 die "neue Abteilung« einrichtete, nämlich den sogenannten Gulag (Lagerhauptverwaltung). Die Gefangenen des Gulag spielten fortan unter anderem eine zentrale Rolle bei industriellen Großbauprojekten wie beispielsweise beim Bau des großen Kanals vom Weißen Meer zur Ostsee, dem Belomorkanal. Dem Projekt kam in den Augen Stalins eine militärisch-strategische Bedeutung bei. Nach der Fertigstellung des Kanals innerhalb von zwei Jahren unter der Führung des stellvertretenden Vorsitzenden der GPU Henrich Jagoda mussten die Gefangenen des Gulag noch Anfang der 1930er-Jahre Zwangsarbeit bei weiteren industriellen Großprojekten leisten - unter anderem beim Bau der Baikal-Amur-Eisenbahn im Fernen Osten oder beim MoskauWolga-Kanal. ${ }^{403}$

401 Vgl. ebd., S. $81 \mathrm{f}$.

402 Ebd., S. 84.

403 Vgl. ebd., S. $118 f$. 